Service social

\title{
Styles de relations et conduites sexuelles d'hommes francophones montréalais qui ont des rapports sexuels avec d'autres hommes
}

\author{
Joseph J. Lévy, André Dupras, Michel Doras, Michel Perreault et Louis-Robert \\ Frigault
}

Volume 45, numéro 1, 1996

Le sexuel et le relationnel

URI : https://id.erudit.org/iderudit/706718ar

DOI : https://doi.org/10.7202/706718ar

Aller au sommaire du numéro

Éditeur(s)

École de service social de l'Université Laval

ISSN

1708-1734 (numérique)

Découvrir la revue

Citer cet article

Lévy, J. J., Dupras, A., Doras, M., Perreault, M. \& Frigault, L.-R. (1996). Styles de relations et conduites sexuelles d'hommes francophones montréalais qui ont des rapports sexuels avec d'autres hommes. Service social, 45(1), 121-135.

https://doi.org/10.7202/706718ar

\section{Résumé de l'article}

Dans le cadre d'une étude réalisée à Montréal, 425 hommes francophones nés au Québec ayant des relations sexuelles avec d'autres hommes ont répondu à un questionnaire à remplir soi-même. Ce questionnaire portait sur leur conception de la sexualité, leurs conduites sexuelles, leurs attitudes à l'égard du condom, leurs rapports de pouvoir et de négociation en situation érotique et leur participation à certaines activités de la communauté gaie. Il ressort que monogamie, non-monogamie ou rapports occasionnels interviennent sur les dimensions précédentes, dessinant des styles de vie relativement différenciés. Ces derniers constituent donc un aspect important pour comprendre la dynamique de la vie amoureuse et sexuelle des hommes ayant des relations sexuelles avec d'autres hommes. 


\title{
Styles de relations et conduites sexuelles d'hommes francophones montréalais qui ont des rapports sexuels avec d'autres hommes
}

\author{
Joseph J. LÉVY \\ Professeur \\ Département de sexologie \\ Université du Québec à Montréal \\ André DUPRAS \\ Professeur \\ Département de sexologie \\ Université du Québec à Montréal \\ Michel DORAIS \\ Professeur associé \\ Département de sociologie \\ Université du Québec à Montréal \\ Michel PerReault \\ Professeur \\ Faculté des sciences infirmières, Université de Montréal \\ Louis-Robert FRIGAULT \\ Doctorant \\ Département d'anthropologie, Université de Montréal
}

Afin d'alléger la lecture de ce texte destiné à des intervenants, les éléments mathématiques et statistiques ont sciemment été réduits à leur plus simple expression. Les auteurs prient le lecteur qui serait intéressé à approfondir ces détails de consulter le rapport original de la recherche, intitulé Styles de vie et comportements sexuels des hommes francophones de Montréal ayant des rapports sexuels avec d'autres hommes. Ce projet a été subventionné par le Conseil québécois de la recherche sociale. 


\section{INTRODUCTION}

Parmi les hommes ayant des relations sexuelles avec des hommes existent une grande diversité d'attitudes, de conduites, de perceptions de soi et des autres. Conduites, orientations et identités homosexuelles ne se recouvrent pas forcément (Welzer-Lang et al., 1994). Les hommes qui ont des relations sexuelles avec d'autres hommes font face, notamment, à une certaine stigmatisation sociale, à une faible reconnaissance de leurs relations de couple, à une carence de modèles sociaux, parfois à des problèmes de santé amplifiés par l'apparition du sida. Ces réalités n'aident en rien leur intégration sociale et leurs relations intimes. Avant d'arriver à adopter un mode de vie épanouissant et adapté à leurs propres besoins, ils ont souvent exploré diverses avenues; les styles de vie à l'intérieur des sous-cultures homosexuelles sont d'ailleurs très diversifiés. L'étude de leurs conduites sexuelles et de leurs styles de vie amoureux constitue donc un moyen privilégié pour mieux connaître le vécu de ces hommes.

Depuis quelques années plusieurs études ont précisément porté sur les conduites sexuelles de cette population, que ce soit aux États-Unis ou en Europe (Donovan et al., 1994; Hospers et Kok, 1995 ; Pollak, 1989 ; Pollak et Schiltz, 1991). Ces recherches ont mis en relief les variations existant dans l'élaboration des pratiques sexuelles, de leurs représentations et des préventions à l'égard du sida. Plus particulièrement, les études qui abordent les styles de relations retiennent trois configurations (Hospers et Kok, 1995): des relations de couple monogame, cohabitant ou non, dont la stabilité peut par ailleurs être problématique (Pollak, 1989), des relations de couple qui s'accompagnent de relations avec d'autres partenaires à l'extérieur de cette dyade, enfin des relations uniquement avec des partenaires occasionnels. Ces styles de relations peuvent être conditionnés par l'âge, le milieu urbain ou rural, le statut socioéconomique, et s'accompagner de conduites préventives différentes. Selon une recherche italienne (Sasse et al., 1991), la pénétration active était rapportée plus fréquemment avec des partenaires stables $(72,9 \%)$ qu'avec des partenaires occasionnels $(63,8 \%)$. La pratique de la pénétration passive présentait une différence encore plus marquée : $64,8 \%$ chez les partenaires stables $v s 51,5 \%$ chez les occasionnels. L'usage du préservatif était aussi plus élevé avec les partenaires occasionnels (72\%) qu'avec les partenaires stables $(48,2 \%)$. La modification des habitudes sexuelles s'avérait plus élevée parmi les répondants ayant des partenaires occasionnels. 
De telles données sont confirmées par de nombreuses autres études menées en Europe et aux États-Unis (Bochow, 1991; Davies et Weatherburn, 1991; Fitzpatrick et al., 1989 et 1990; Hays et al., 1990; McKusick et al., 1985 ; Stall et al., 1992; Valdisseri et al., 1988). Elles montrent en outre que les hommes qui sont dans une relation stable utilisent moins le condom que ceux qui ont des relations avec des partenaires occasionnels. Par exemple Doornbos (1992) constate que $9 \%$ des hommes ayant des relations anales avec des partenaires occasionnels n'utilisent pas toujours le condom, alors que $28 \%$ de ceux qui ont à la fois un partenaire régulier et des partenaires occasionnels ne se protègent pas toujours. Ce pourcentage grimpe à $47 \%$ chez les partenaires exclusifs. Les recherches canadiennes menées par Myers et al. (1993) confirment cette tendance. Il en irait de même au Québec selon Godin et al. (1994), Myers et al. (1993), Lafontaine (1995) et Pleau (1989).

Nous avons voulu dans la présente étude raffiner notre compréhension des liens qui peuvent exister entre les différents types de relations érotiques (monogame, non monogame, occasionnelle) et certaines attitudes et conduites sociosexuelles. Nous avons surtout voulu dégager de nouvelles pistes pour comprendre l'adoption ou non de comportements sexuels sécuritaires par les hommes de la population étudiée, en tenant compte de leur conception de la sexualité et de la nature de leurs rapports érotiques.

\section{MÉTHODOLOGIE}

\section{Échantillon}

Cette étude est basée sur un échantillon non probabiliste d'hommes ayant des relations sexuelles avec d'autres hommes. Ces 425 répondants sont tous nés au Québec, ils sont francophones et sexuellement actifs. Ils demeurent dans la région du Montréal métropolitain. Ils ont répondu à nos questions au cours de l'année 1994. Deux techniques de recrutement ont été retenues. Le plus grand nombre des sujets a été rejoint par l'intermédiaire d'organismes communautaires $(40,9 \%)$, d'établissements commerciaux gais $(21,6 \%)$, de saunas gais $(10,6 \%)$ et de cliniques médicales desservant cette population (6,4\%). Par ailleurs, 20,5\% des répondants ont été rejoints par une méthode dite de boule de neige (diffusion de questionnaires entre amis et connaissances), destinée à rejoindre des hommes qui ne s'identifient pas forcément à la communauté gaie ou qui ne la fréquentent pas. 
La distribution des âges se répartit de la façon suivante: $16,2 \%$ âgés de moins de 25 ans, $23,8 \%$ entre 26 et 30 ans, $36,5 \%$ entre 31 et 40 ans, $23,5 \%$ âgés de 41 ans et plus. La majorité sont célibataires $(81,6 \%) ; 46,6 \%$ des répondants vivent seuls, $24,5 \%$ en couple, $16,7 \%$ en colocation, $8 \%$ avec un membre de leur famille, $6 \%$ avec des amis et presque $1 \%$ avec une épouse. Sur le plan de la scolarité, $47,1 \%$ ont terminé des études universitaires, 26,8\% des études collégiales, 26,1\% des études primaires ou secondaires. En ce qui concerne l'emploi, 63,8\% ont un travail à plein temps, $19,1 \%$ à temps partiel et $17,2 \%$ déclarent ne pas avoir d'emploi. Pour ce qui est du revenu annuel, 34,9\% gagnent moins de $20000 \$, 42,1 \%$ ont un revenu qui se situe entre $20000 \$$ et $40000 \$$ et $21,8 \%$ gagnent plus de $40000 \$$. Quant à la croyance et à la pratique religieuses, 96,2\% déclarent avoir été élevés dans le catholicisme. 38,4\% se définissent comme croyants, $34,8 \%$ comme peu croyants et $26,6 \%$ comme non croyants. Une proportion de $11 \%$ assistent au moins une fois par mois à un office religieux.

Parmi les répondants, 29,4\% rapportent avoir eu un partenaire régulier et exclusif au cours des six derniers mois, 28,6\% ont eu un partenaire régulier mais non exclusif, alors que $42 \%$ n'ont eu que des partenaires occasionnels. Aucune différence n'apparaît entre ces catégories quant aux revenus personnels, à la scolarité, aux croyances ou aux pratiques religieuses. Ces données correspondent assez à celles de Pollak et Schiltz (1991), alors qu'elles diffèrent du profil des répondants montréalais de Myers et al. (1993) : 61,5\% n'avaient pas de relation stable, $17 \%$ avaient une relation non monogame et $18,3 \%$ une relation monogame.

Un peu plus de $13 \%$ des répondants se déclarent séropositifs, ce qui est un taux plus bas que celui rapporté par la recherche de Myers et al. (1993) pour la région de Montréal (20,5\%).

\section{Questionnaire}

À remplir soi-même, le questionnaire comportait plusieurs sections: variables sociodémographiques, participation à la communauté gaie, perception de la sexualité et de l'érotisme, contrôle et pouvoir personnels en contextes sexuels, perception de son efficacité à contrer les risques de contracter le sida, pratiques sexuelles, perception du condom et du sida, type de rapports amoureux, facteurs de risque lors de relations sexuelles (par exemple consommation de drogues et d'alcool). 
Pour les questions relatives à la participation à la communauté gaie nous nous sommes inspirés de Myers et al. (1993). Pour les questions concernant les rapports de pouvoir, nous avons emprunté à Brown (1992) les concepts d'influence dans le contexte sexuel (contribution à la prise de décision), de communication sexuelle (transmettre des informations sur ses désirs et besoins) et de pression en contexte sexuel (niveau de coercition exercé lors de relations sexuelles). Pour jauger la recherche de sensations, les énoncés ont été formulés à partir de l'échelle de Zuckerman (1979) et de Zuckerman et Myers (1983). Pour évaluer les attitudes à l'égard de l'utilisation du préservatif, nous avons construit des énoncés à partir d'un ensemble de thèmes accolés à la perception, positive ou négative, du condom dans la littérature sur le sujet. Les analyses factorielles montrent l'existence de deux facteurs significatifs: le condom comme obstacle à la relation sexuelle ou comme barrière efficace.

En ce qui concerne la mesure de l'efficacité personnelle dans la protection contre le sida, nous avons utilisé Thabet et al. (1995). Les énoncés ayant trait au contrôle des pulsions sexuelles ont été empruntés à la recherche de Exner et al. (1992). Ce contrôle est défini par la capacité qu'a un individu de s'abstenir ou d'éviter rationnellement certaines pratiques sexuelles, notamment lorsqu'elles comportent des risques de contracter le sida. Nous avons aussi interrogé les répondants sur leurs tests de dépistage du VIH et sur les résultats obtenus à ces tests, sur la peur d'être infectés et sur les changements d'habitudes qui en ont découlé.

Les données recueillies par cette enquête ont été analysées à l'aide du progiciel SPSS 4.0 pour UNIX. En plus des analyses bivariées, des analyses de régression logistique ont été faites. Le présent article mettra l'accent sur les différences de conduites sociosexuelles existant entre les individus selon qu'ils sont engagés dans des relations monogames, non monogames ou occasionnelles.

\section{RÉSULTATS}

\section{Participation à des activités de la communauté gaie}

On note que les moins de 25 ans et ceux qui n'ont que des partenaires occasionnels fréquentent davantage les bars gais. A l'inverse, ce sont les hommes plus âgés de l'échantillon qui 
fréquentent le plus les saunas, quoique, là encore, ceux qui n'ont pas de partenaires réguliers sont les plus enclins à les fréquenter assidûment.

Ceux qui sont dans des relations stables sont moins portés à participer aux activités de la communauté gaie, tandis que ceux qui n'ont que des partenaires occasionnels sont les plus actifs de ce côté. La majorité des répondants ont fréquenté au moins une fois un bar gai au cours des six derniers mois $(13,9 \%$ ne les ont pas fréquentés du tout). Seulement $5,4 \%$ de ceux qui ont un partenaire régulier exclusif ont fréquenté les saunas régulièrement au cours des six derniers mois ; $33,4 \%$ de ceux qui ont un partenaire régulier dans une relation non exclusive et $26,9 \%$ de ceux qui n'ont que des partenaires occasionnels ont fait de même. Près du quart des répondants ont eu recours à des lignes de rencontre téléphoniques gaies au cours des six derniers mois. La fréquence d'utilisation est indépendante de l'âge et du type de relation. Enfin, la lecture des journaux gais constitue l'activité la plus courante: plus de $95 \%$ des répondants ont consulté journaux ou magazines gais au cours des six derniers mois.

\section{Conception de la sexualité et rapports de pouvoir}

En moyenne, les répondants adoptent une conception de la sexualité qui se rapproche davantage du pôle irrationnel (échappant au contrôle ou aux décisions de l'individu) que du pôle rationnel (pouvant être décidé ou contrôlé). Cette caractéristique ne varie pas selon le type de relations entretenues. Des différences significatives apparaissent cependant dans les relations de pouvoir entre partenaires. Ainsi, le choix de l'endroit où ont lieu les relations sexuelles est moins transitif chez les répondants qui ont des partenaires occasionnels uniquement. Plus les répondants sont jeunes, moins ils ont tendance à choisir le lieu où se déroulent les relations entre partenaires.

La réciprocité de l'influence manifestée dans la décision d'avoir des relations sexuelles serait la plus faible parmi ceux qui n'ont que des partenaires occasionnels et la plus forte parmi ceux qui sont en relation non monogame, ceux qui vivent des relations stables se situant entre les deux. Enfin, ceux qui sont en relation stable sont les moins vulnérables à la pression en contexte sexuel. Dans le même ordre d'idées, plus les répondants sont jeunes, plus ils rapportent avoir eu des relations sexuelles alors qu'ils ne le voulaient pas au cours des six derniers mois: 
$32,2 \%$ chez les moins de 25 ans, 30,7\% chez les 26 à 30 ans, $26 \%$ chez les 31 à 40 ans et $16,2 \%$ chez les 41 ans et plus. Inversement, $14,5 \%$ des répondants auraient eu des rapports sexuels avec quelqu'un qui ne voulait pas vraiment en avoir.

Le contrôle de ses pulsions sexuelles varie selon le type de relation vécue. Ceux qui ont un partenaire régulier et sont monogames auraient un meilleur contrôle que ceux qui ont des partenaires occasionnels uniquement ou qui sont non monogames. Il en va de même pour la quête de sensations: elle est la moins élevée chez les monogames, ceux-ci étant suivis par les «occasionnels», puis par les non-monogames. Par ailleurs - ce qui pourra sembler paradoxal-, ceux qui ont un partenaire régulier monogame estiment plus excitantes leurs relations sexuelles que ceux qui sont non monogames ou qui ont des partenaires occasionnels uniquement.

\section{Attitudes à l'égard du condom et modifications des pratiques sexuelles}

Selon nos répondants le plaisir retiré des activités sexuelles ne serait guère affecté par l'usage du condom, que ce soit comme obstacle ou comme stimulant. L'utilisation du condom nuit toutefois moins au plaisir sexuel des monogames que des nonmonogames et de ceux qui n'ont que des partenaires occasionnels. Par ailleurs, plus longue est la période de monogamie, plus faible est la fréquence d'utilisation du condom. En revanche, la perception de la fonction de prévention du condom est assez bien intégrée chez tous les répondants de cette étude, quel que soit le type de relation. Les partenaires réguliers utilisent le condom dans $60 \%$ de leurs relations anales, que celles-ci soient actives ou passives. Dans le cas des partenaires occasionnels ce pourcentage se situe au-dessus de $90 \%$.

La peur du sida et l'évaluation à la baisse des probabilités d'être infecté par le VIH sont associées à une plus forte utilisation du condom. À l'inverse, le fait de subir des pressions dans le contexte sexuel, de rechercher des sensations fortes ou le fait de considérer la sexualité comme irrationnelle sont liés à une plus faible utilisation du condom. De même, une évaluation à la hausse des probabilités d'être infecté par le VIH, le sentiment de faible contrôle sur ses pratiques sexuelles et une faible impression d'auto-efficacité personnelle sont corrélés à une plus faible utilisation du condom. 


\section{Conduites sexuelles}

On constate peu de différences dans les conduites sexuelles entre les groupes de monogames, de non-monogames et de partenaires occasionnels. L'âge des premières relations sexuelles se situe en moyenne à 17,8 ans pour les relations oro-génitales et 20,9 ans pour les relations anales. Les relations anales actives sont plus fréquentes chez les non-monogames ( $71,7 \%)$ que chez les monogames $(46,9 \%)$ et les partenaires occasionnels $(46,8 \%)$. Il en va de même pour les relations anales passives : $63,3 \%$ des non-monogames en ont, alors que ce pourcentage est de $45,3 \%$ chez les monogames et de $36,2 \%$ chez les partenaires occasionnels. Les relations anales tendent à diminuer avec l'âge. Notons que chez les non-monogames et les occasionnels le nombre moyen de partenaires dans les six mois précédant l'enquête était de dix.

Des différences apparaissent dans l'utilisation du condom. Les répondants monogames l'utilisent moins lors de relations anales que les non-monogames ne le font avec leur partenaire régulier. Même lorsqu'ils ont consommé drogue et alcool, ceux qui ont des partenaires occasionnels se protègent $(78,7 \%$ lors de relations anales actives et $86,3 \%$ lors de relations anales passives chez les «occasionnels » contre $58,4 \%$ et $47,3 \%$ chez ceux qui ont des partenaires réguliers).

\section{Tests de dépistage du VIH et attitudes à l'égard du sida}

La majorité des hommes interrogés, soit 76,3\%, avaient déjà passé un test afin de savoir s'ils étaient porteurs du VIH. Parmi ceux qui n'ont pas passé ce test, le tiers affirment avoir l'intention de le faire dans un avenir rapproché.

Parmi les répondants, $54 \%$ avaient déjà contracté au moins une MTS. C'est parmi ceux qui n'ont eu que des partenaires occasionnels que ce pourcentage est le plus élevé $(61,6 \%)$, suivis par les non-monogames $(55,5 \%)$ et les monogames $(44,7 \%)$. La grande majorité des répondants, soit 94,1\%, affirment avoir modifié leurs conduites sexuelles depuis l'apparition du sida. Par conséquent ils perçoivent positivement leur efficacité à contrer la transmission du VIH. Ainsi, 48,8\% des hommes qui ont un partenaire régulier dans une relation monogame jugent que leurs pratiques sexuelles ne comportent aucun risque de contamination par le VIH. Ce pourcentage tombe à 38,3\% chez ceux qui n'ont que des partenaires occasionnels et à seulement 
$12,9 \%$ chez ceux qui ont un partenaire régulier dans une relation non monogame.

Seulement $17,2 \%$ des répondants affirment ne pas avoir peur du tout de devenir infectés par le VIH. Les moins de 25 ans sont ceux qui ressentent le plus cette peur. Uniquement 5,9\% des hommes interrogés disent n'avoir aucunement changé leurs habitudes sexuelles depuis l'apparition du sida.

\section{DISCUSSION}

Ces données vont à l'encontre du préjugé selon lequel les hommes homosexuels préféreraient les partenaires occasionnels aux relations stables. En effet, plus de $58 \%$ des hommes de notre échantillon vivent ou ont récemment vécu une relation de couple, qu'elle soit monogame $(29,5 \%)$ ou non monogame $(28,6 \%)$. Bell et Weinberg (1978) constataient il y a près de vingt ans la même tendance : $61 \%$ de leurs répondants affirmaient que de vivre avec un partenaire était assez important ou très important pour eux. L'avènement du sida donne un nouvel attrait à la vie de couple et, en corollaire, signifie une baisse d'intérêt à l'endroit des activités avec des partenaires occasionnels (Dupras, 1994 ; Pollak et Schiltz, 1991). Dans le même sens, Bringle (1995) rapporte que les hommes gais mentionnent vivre des relations de couple plus durables dans les années 1990 qu'au tout début des années 1980.

Les styles de relations entre partenaires semblent induire des niveaux de participation différents aux activités de la communauté gaie. Ainsi, bien que les bars, saunas ou autres lieux de rencontre constituent à la fois des lieux de socialisation et de flirt, les couples monogames les fréquentent beaucoup moins: $50 \% \mathrm{y}$ vont très rarement sinon jamais, alors que près de $70 \%$ des non-monogames et près de $75 \%$ des non-engagés les fréquentent plus d'une fois par mois. Les non-monogames et ceux qui ne sont pas en couple s'intéressent davantage aux activités sociales en milieu gai. Les données de notre enquête révèlent également des rapports de pouvoir variables selon le style de relations. Si les hommes ayant des relations avec d'autres hommes souhaitent un partage égal du pouvoir entre partenaires (Pepleau, 1991), tous ne voient pas leur désir exaucé, en particulier les plus jeunes.

Les sujets vivant en couple monogame rapportent dans $67,3 \%$ des cas parler régulièrement ensemble de leur sexualité ; 
$54,8 \%$ des non-monogames et 33,9\% des «occasionnels» font de même. Kollock et al. (1985) ont toutefois constaté que cette communication n'est pas forcément transitive: un partenaire peut tenter d'en contrôler un autre par ses questions et commentaires. Ces rapports inégalitaires constituent souvent une source de conflit et d'insatisfaction, voire un facteur de rupture (Blumstein et Schwartz, 1983 ; Kuredek, 1994). Ce sont toutefois les sujets vivant en couple ouvert ou n'ayant que des relations occasionnelles qui cèdent le plus aux pressions d'autrui pour avoir des relations sexuelles.

En conformité avec nos propres données, certaines études récentes (Hickson et al., 1994 ; Kalichman et Rompa, 1995) vont jusqu'à affirmer que plus du quart des hommes gais se seraient sentis forcés d'avoir des relations sexuelles contre leur consentement ou n'auraient pu ou su repousser de telles avances. La majorité de ces activités ont pris la forme de pénétrations anales non protégées, ce qui ne va pas sans risques au regard de l'infection au VIH.

Contrairement à la croyance populaire, le niveau d'excitation sexuelle le plus satisfaisant est vécu par ceux qui entretiennent une relation monogame. La qualité de la relation semble alors l'emporter sur la quantité. Hendrick et Hendrick (1988) avaient déjà noté chez les collégiens en amour qu'ils cherchaient moins de "sensations fortes", alors que Fischer et Misovich (1990) constataient que les hommes qui avaient le plus de partenaires sexuels étaient ceux qui recherchaient le plus ces «sensations fortes". Ils sont parfois prêts à expérimenter des "pratiques à risques" afin de se satisfaire (Kalichman et al., 1994).

Motivations et valeurs prônées par les partenaires de même sexe semblent influencer grandement leurs conduites sexuelles. Par exemple, l'exclusivité induit un style de vie relativement différent de la non-exclusivité ou du non-engagement relationnel. Les non-monogames et les non-engagés participent en effet davantage à l'ensemble des activités offertes par le milieu gai, qu'elles soient connotées sexuellement ou non. On notera que Pollak et Schiltz (1991) divisent les couples non monogames entre ceux qui ont une faible promiscuité et ceux qui ont une forte promiscuité à l'extérieur de leur relation. Shernoff (1995) fait remarquer que les «couples fermés » peuvent être subdivisés entre ceux qui sont peu ou très motivés par le sexe, que les «couples ouverts » peuvent être subdivisés entre ceux qui ont 
des relations secrètes ou révélées et qu'il existe aussi des relations à trois partenaires.

Il est frappant que plus de la moitié des répondants aient eu au moins une MTS, bien qu'ils soient nombreux à croire leur stratégie préventive efficace. Si l'on compare le nombre de personnes ayant été dépistées pour le VIH $(76,3 \%)$ avec les résultats de l'enquête de Myers et al. (1993) (53\%), on peut déduire que le dépistage est devenu un élément de plus en plus intégré dans la vie des hommes ayant des relations avec d'autres hommes.

\section{CONCLUSION}

Cette étude sur les hommes francophones montréalais qui ont des relations avec d'autres hommes montre que ces répondants présentent à la fois des convergences et des divergences selon leur style de vie monogame, non monogame ou ouvert. Elle permet aussi d'effectuer des distinctions quant à l'âge en ce qui concerne la fréquentation des bars et des saunas, et les rapports de pouvoir inégaux : les plus jeunes étant ceux qui choisissent le moins les lieux où ils ont des relations sexuelles et, plus encore, qui subissent le plus des relations sexuelles non vraiment désirées. Les intervenants psychosociaux et communautaires devraient donc privilégier l'action éducative et le soutien auprès des jeunes hommes de moins de vingt-cinq ans qui ont des relations avec d'autres hommes: ce sont les plus vulnérables en ce qui concerne les pratiques à risques de transmission du VIH-sida, puisqu'ils sont les plus enclins à subir l'influence de leurs partenaires uniquement. Comme ils ont souvent appris davantage à camoufler leurs sentiments et volontés qu'à les révéler - à cause de la stigmatisation sociale de leurs désirs -, ils n'ont pas toujours appris à affirmer leurs limites et leurs besoins. Cela constitue une dangereuse carence quand il est question aujourd'hui de relations sexuelles non voulues ou mal protégées. Les partenaires en relations non monogames se retrouvent aussi dans une situation parfois inconfortable: moins de $13 \%$ considèrent leurs rapports intimes comme étant sans risque, ce qui constitue le pourcentage le plus bas de cette enquête. Quel impact cette inquiétude a-t-elle sur leur relation? La question mériterait d'être posée et la recherche à ce sujet approfondie.

Les intervenants psychosociaux doivent prendre davantage conscience des vulnérabilités associées à l'âge et aux styles de 
relations quand il est question de rapports sexuels entre hommes. Il importe aussi qu'ils reconnaissent la nécessité de procurer aux jeunes hommes gais ou bisexuels des conditions de vie faisant en sorte qu'ils puissent s'affirmer sereinement et négocier d'égal à égal leurs rapports amoureux et sexuels. En somme, l'ensemble de ces résultats confirme l'importance de cibler plus finement certaines sous-populations selon leur âge et leur style de vie amoureux en ce qui concerne la prévention et l'éducation en matière de VIH-sida.

\section{Références bibliographiques}

ANTIL, T. (1995). "Les hommes ayant des relations sexuelles avec d'autres hommes. Un bilan des études québécoises », Sida-Presse, vol. $5, \mathrm{n}^{\circ} 2: 7-11$.

BELL, A.P. et M.S. Weinberg (1978). Homosexualités. Paris: Albin Michel.

Blumstein, P. et P. SCHWARTZ (1983). American Couples. New York: Willam Morrow.

Bochow, M. (1991). «Le safer sex: une discussion sans fin», dans M. Pollak, R. Mendes-Leite et J. Van Dem Borghe (dir.), Homosexualités et sida, p. 117-131. Paris : Cahiers Gai-Kitsch-Camp.

BRINGLE, R.G. (1995). «Sexual jealousy in the relationships of homosexual and heterosexual men: 1980 and $1992 »$, Personal Relationships, vol. $2: 313-325$.

BROWN, J.A. (1992). Safer sex practices: Gender, power and risk in a college student population. Storrs, Conn.: Université du Connecticut, UMI. Thèse de doctorat non publiée.

DAVIES, P. et P. WEATHERBURN (1991). "Towards a general model of sexual negociation », dans P. Aggleton, G. Hart et P. Davies (dir.), AIDS : Responses, interventions and care, p. 111-115. London: Falmer Press.

DEENEN, A.A., L. GIJS et L.X. VAN NAERSSEN (1995). «Thirty-five years of research into gay relationships », Journal of Psychology and Human Sexuality, vol. 7, $\mathrm{n}^{\mathrm{o}} 4: 19-39$.

De Wit, J.B.F., E.M.N. De Vroome, T.G.M. Sandfort, G.J.P. Van GRIENSVEN, R.A. COUTINHO et R.A.P. TIELMAN (1992). "Safe sexual practices not reliably maintained by homosexual men", American Journal of Public Health, vol. 82, $\mathrm{n}^{\circ} 4$ : 615-616.

Donovan, C., C. Mearns, R. MCEwan et N. Sugden (1994). «A review of the HIV-related sexual behaviour of gay men and men who have sex with men», AIDS Care, vol. 6, $\mathrm{n}^{\circ} 5: 605-617$ 
Doornbos, R. (1992). Safe and unsafe sex between men. Utrecht, The Netherlands: University of Utrecht, Department of Gay and Lesbian Studies.

DUPRAS, A. (1994). «L'impact du sida sur la vie sexuelle des hommes homosexuels : résultats d'une enquête menée à Montréal », Revue sexologique, vol. $1, \mathrm{n}^{\circ} 2: 54-74$.

EXNer, T.M., H.F.L. MEYER-BAhlbURG et A.A. EHRHARDT (1992). "Sexual self control as a mediator of high risk sexual behavior in a New York City cohort of HIV+ and HIV- gay men », The Journal of Sex Research, vol. 29, $\mathrm{n}^{\circ} 3: 389-406$.

Fisher, J.D. et S.J. MisOviCH (1990). "Social influence and AIDSpreventive behavior», dans J. Edwards, R.S. Tindale, L. Health et E.J. Posavac (dir.), Social influence processes and prevention, p. 39-70. New York: Plenum.

FitzPATRick, R., M. Boulton, G. HART, J. DAwson et J. MCLEAN (1989). "High risk sexual behaviour and condom use in a sample of homosexual and bisexual men », Health Trends, vol. 21 : 76-79.

FitzPATRiCK, R., J. MCleAn, M. BOUlton, G. HART et J. DAWSON (1990). "Variation in sexual behaviour in gay men", dans P. Aggleton, P. Davis et G. Hart (dir.), AIDS : Individual, cultural, and policy dimensions, p. 121-132. London : Falmer Press.

Godin, G., J. CARSLEY, K. MORRISON et R. BRADET (1994). «Les relations anales et l'utilisation du condom chez les hommes ayant des relations sexuelles avec d'autres hommes: résultats de l'enquête québécoise ", Revue sexologique, vol. 2, nº 1:1-17.

HAYs, R.B., S.M. KegEles et T.J. COATES (1990). "High HIV risk-taking among young gay men », AIDS, vol. 4, n 9 : 901-907.

HENDRICK, C. et S.S. HENDRICK (1988). "Lovers wear rose colored glasses », Journal of Social and Personal Relationships, vol. 5, $\mathrm{n}^{\circ} 2$ : 161-183.

Hickson, F.C.I., P.M. Davies, A.J. Hunt, P. WeAtherburn, T.J. MCMANus et A.P.M. COXON (1994). "Gay men as victims of nonconsensual sex », Archives of Sexual Behavior, vol. 23, n $3: 281-294$.

Hospers, H.J. et G. KOK (1995). «Determinants of safe and risk-taking sexual behavior among gay men : A review », AIDS Education and Prevention, vol. 7, $\mathrm{n}^{\mathrm{o}}$ 1: 74-96.

KALiCHMAN, S.C., J.R. JOHNSON, V. AdAIR, D. ROMPA, K. Multhauf et J.A. KELlY (1994). "Sexual sensation seeking : scale development and predicting AIDS-risk behavior among homosexually active men », Journal of Personality Assessment, vol. 62, n ${ }^{\circ} 3$ : 385-397.

KALICHMAN, S. et C.D. ROMPA (1995). "Sexually coerced and noncoerced gay and bisexual men: Factors relevant to risk for human immunodeficiency virus (HIV) infection", The Journal of Sex Research, vol. 32, $\mathrm{n}^{\circ} 1: 45-50$.

Kollock, P., P. Blumstein et P. SCHWARTz (1985). «Sex and power in interaction: Conversational privileges and duties ", American Sociological Review, $50:$ 34-46. 
KURDEK, L.A. (1994). "Areas of conflict for gay, lesbian, and heterosexual couples: What couples argue about influences relationship satisfaction », Journal of Marriage and the Family, 56: 923-934.

LAFONTAINE, Y. (1995). "Résultats du sondage sur la sexualité des gais et leurs modes de vie », Fugues, avril, p. 52-76.

MCKusick, L., J.A. Wiley, T.J. CoATes, R. Stall, G. SAika, S. Morin, K. Charles, W. Horston et M.A. ConAnt (1985). "Reported changes in the sexual behaviour of men at risk for AIDS in San Francisco, 1982-1984, the AIDS Behavioural Research Project», Public Health Reports, vol. 100, $\mathrm{n}^{\circ} 6$ : 622-629.

Myers, T., G. Godin, L. Calzavera, J. LAmbert et D. Locker (1993). L'enquête canadienne sur l'infection à VIH menée auprès des hommes gais et bisexuels : au masculin. Ottawa : Société canadienne du sida.

PEPLAU, L.A. (1991). «Lesbian and gay relationship», dans J.C. Gonsiorek et J.D. Weinrich (dir.), Homosexuality. Research implications for public policy, p. 177-196. Newbury Park: Sage Publications.

PEPLAU, L.A. et S.D. COCHRAN (1988). «Value orientation in the intimate relationships of gay men », dans J.P. De Cecco (dir.) : Gay relationship, p. 195-216. New York: The Haworth, Inc.

Pleau, M. (1989). "Sondage. Que font les gais ?», RG, juin, p. 23-27.

Pollak, M. (1989). «Les homos et le sida», Gai Pied Hebdo, vol. 395 : 52-55.

POllaK, M. et M.A. SCHILTz (1991). Six années d'enquête sur les homoet bisexuels masculins face au sida. Paris: École des Hautes Études en sciences sociales. Rapport de recherche.

SANTÉ CANADA (1994). Lignes directrices nationales pour l'éducation en matière de santé sexuelle. Ottawa: ministère de la Santé nationale et du Bien-être social.

Sasse, H., A. Bigagli, F. Chiarotti, P. Martucci, D. Greco et F. Grillini (1991). "Pratiques homosexuelles avec des partenaires stables et partenaires occasionnels chez les homosexuels en Italie », dans M. Pollak, R. Mendes Leite et J. Van Dem Borghe (dir.), Homosexualités et sida, p. 69-81. Paris: Cahiers Gai-Kitsch-Camp.

SCHWARTZ, S.H. (1992). "Universal in the content and structure of values: Theoretical advances and empirical tests in 20 countries », dans M.P. Zanna (dir.), Advances in experimental social psychology, p. 1-65. New York: Academic Press.

SCHERNOFF, M. (1995). "Male couples and their relationship styles", Journal of Gay and Lesbian Social Services, vol. 2, $\mathrm{n}^{\circ} 2$ : 43-57.

Stall. R., D. BArret, L. Bye, J. Catania, C. FrutChey, J. Henne, G. Lemp et J. PAUL (1992). "A comparison of younger and older gay men's HIV risk taking behaviors: The communication technologies 1989 cross-sectional survey», Journal of Acquired Immune Deficiency Syndromes, vol. 5, $\mathrm{n}^{\circ} 7: 682-687$.

ThabeT, C., J. OTIS et A. Dupras (1995). «Les croyances des jeunes à l'égard de l'utilisation des distributeurs de préservatifs à l'école : utilité de la théorie sociale cognitive», Revue sexologique, vol. 3, $\mathrm{n}^{\circ} 2: 143-167$. 
VAldisseri, R.O., D. Lyter, L.C. Leviton, C.M. Callahan, L.A. Kingsley et C.R. RINALDO (1988). "Variables influencing condom use in a cohort of gay and bisexual men », American Journal of Public Health, vol. 78, $\mathrm{n}^{\circ} 7: 801-805$.

Welzer-LANG, D., P. Dutey et M. Dorais (1994). La Peur de l'autre en soi : du sexisme à l'homophobie. Montréal : VLB éditeur.

ZUCKERMAN, M. (1979). Sensation seeking: Beyond the optimal level of arousal. Hillsdale, N.J. : Lawrence Erlbaum Assoc.

ZUCKERMAN, M. et P.L. MYERS (1983). "Sensation seeking in homosexual and heterosexual males», Archives of Sexual Behavior, vol. $12, \mathrm{n}^{\circ} 4: 347-356$. 